\title{
Sparse Representation based Satellite Image Restoration Using Adaptive Reciprocal Cell
}

\author{
Yanfei $\mathrm{He}^{1}$, Jianwei Zhang ${ }^{1}$, Shunfeng Wang ${ }^{1}$, Yuhui Zheng ${ }^{2}$, Jin Wang ${ }^{2}$ and Yunjie \\ Chen $^{1}$ \\ ${ }^{1}$ Department of Math and Statistic, Nanjing University of Information \\ Science \& Technology, Nanjing 210044, China \\ ${ }^{2}$ School of Computer \& Software, Nanjing University of Information \\ Science \& Technology, Nanjing 210044, China \\ Abstract
}

\begin{abstract}
Recently, an emerging method called image sparse representation has attracted more attentions. The method has been proved to be effective in various image processing applications. It is important to note that few sparse representation methods fail to analyze the aliasing in satellite image restoration. fo address the problem, firstly, we employ adaptive reciprocal cell as a image quality estimation tool. Which can analyze the satellite image degradation factors including aliasing, blur and noise. Then, with the help of the powerful tool, the estimation about the satellite Image quality is introduced into the sparse representation model. Experiment lestilts show thav our method can produce good quality restored results.
\end{abstract}

Keywords: Image Restoration, Sparse Representation, Aliasing

\section{Introduction}

In earth observation, sometines there are no other images about the scene of interest but a single satellite image, usually corrupted by aliasing, blur and noise. Therefore, the image needs to be processed to better) reflect its radiometric and geometric quality. This process is called satellite image restoration. Its goal is reconstruction or recovery of the degraded image using a prior knowledge of image degradation process [1-3].

Regularly, the discrete degradation model can be represented by

$$
I=\Delta_{\Gamma} \cdot(h * f)+n
$$

where $T$ is the observed (measured) image. $f$ is the natural scene, defined on a continuous supporta bounded set included in $\left.R^{2}\right)$. $n$ is the additive Gaussian white noise. $(h * f)$ is the convolution product of $f$ by the point-spread function $h$, which is normalized, positive and symmetric with respect to the $\mathrm{x}$ and $\mathrm{y}$ axes. The Fourier transform of $h$ is called the MTF (Modulation Transfer Function). Actually, The effect of the imperfect optical system is characterized by MTF, which is similar to a low-pass filter leading to blurred appearance. $\Delta_{\Gamma}$ is the sampling comb composed of delta-functions $\delta_{i j}$,

$$
\Delta_{\Gamma}=\sum_{(i, j) \in \Gamma} \delta_{i j}
$$


where $\Gamma$ is the sampling grid. It represents the geometry of the array of sensors, which are assumed to be distributed on a regular grid:

$$
\Gamma=\left\{n_{1} e_{1}+n_{2} e_{2}: n_{1}, n_{2} \in \mathrm{Z}\right\}
$$

where $\left\{e_{1}, e_{2}\right\}$ is a basis of $R^{2}$. For square sampling grid $\Gamma_{4}, e_{1}=(1,0)^{T}$, and $e_{2}=(0,1)^{T}$. since $\left\langle\delta_{i j}, f\right\rangle=\int f(x) \delta_{i j} d x=f_{i j}$, sampling on $\Gamma_{4}$ can be expressed as simply multiplying by $\Delta_{\Gamma_{4}}$.

The restoration of satellite image is an ill-posed inverse problem. It's well-known that direct inversion of (1) leads to unacceptable noise amplification. Therefore it is natural to address the limitations of direct methods by using advanced nonlinear regurarization techniques which regularize the inversion problem so that the observed inage can be recovered without amplifying the noise. Some related, wavelet-based satellite image restoration methods have been suggested in the literature [4-6], which can be summarized by the following two steps: deblurring by a pseudo-inverse filter, and then denoising done in a wavelet basis, by thresholding the noisy coefficients. Mainly, the differences between the two-step methods centered on the denoising are sparse representation algorithms [7-9]. Even if the two-step methods can resolve the denoising problem, they would be impractical. Since the optical system would be moving during capture, so that it would produce motion blurs which is invertible. It means that we canty even carrout the first step actually, otherwise ringing artifacts would arise.

F. Malgouyre and F. Guichard 10 hlave also observed the limitations of the wavelet-based methods and suggested to deblur satellite image using variational methods. The variational image resoration methods haye been widely studied. Among them, the total variation (TV) regularization [11, 12] has become a popular method known for preserving discontinuities. Recently, an emerging method called sparse representation of image has attracted lots of attention $[15,16]$. This theory has been proved to be effective in various application, such as image super-resortion [17], in painting [19], classification and image compression [13, 14].

In this work we present adaptive reciprocal cell based image restoration which uses the advanced spare and redundant representation technology. The adaptive reciprocal cell is employed to analyze the degradation factors, such as blurring, noise and aliasing. Then, we introduce the analysis result into the sparse representation model.

\section{Proposed Method}

\subsection{Adaptive Reciprocal Cell}

According to signal sampling theory, (1) can be rewritten by Fourier transform:

$$
\left\{\begin{array}{l}
\tilde{I}(\tilde{X})=\frac{1}{\left|\operatorname{det} V_{s}\right|} \sum_{k} \tilde{f}\left(\tilde{n}-U_{a} k\right) \\
U_{a}^{T} V_{s}=E
\end{array}\right.
$$

where $U_{a}$ is the reciprocal cell which reflects the sampling period of satellite imaging system.

Based on the (4), [18] proposed the adaptive reciprocal cell method and used it to analyze the efficiency of various imaging system. This method can describe the aliasing in various imaging system, and is a new way of measuring the effective resolution of an image acquisition system. 


\subsection{Adaptive Reciprocal Cell based Sparse Representation Model}

The traditional TV regularization model $[20,21]$ is effective in filtering the noise but tends to smooth the image, especially the image structure. This is due to the piecewise smoothing constraint. In recent years, the sparse representation related methods have achieved promising restoration results [22-24].Because of the high dimensionality of image, sparse representation method focuses on small patches of natural images. So the whole image is usually divided into image patches. Each image patch is processed independently, and the final result image is produced by stitching and averaging the patches.

The sparse representation model assume that an image patch $f^{p}$ can be approximately represented via a vector $\alpha$ over a dictionary $\phi \in R^{n \times K}$ (each column in $\phi$ is called an atom). Image patch can be approximately represented as:

$$
f^{p} \approx \phi \alpha, \quad \text { s.t. } \quad\|\alpha\|_{0} \leq T<
$$

where \|\|$_{0}$ is a pseudo norm that counts the number or nonzero items in vector $\alpha$. (5) indicates that the sparse coding of $f^{p}$ can be calculated by solving the $t_{0}$ mininization problem.

As the $l_{0}$ minimization problem is an NP problem, itos often resolved by $l_{1}$ problem which is convex. The related formulation is as follow:

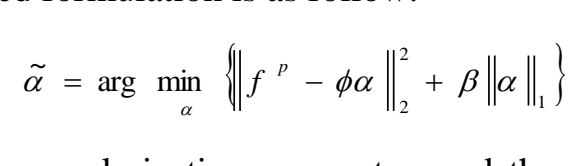

where constant $\beta$ is the regularization parameter, and the second term is sparse coding which is the sparse approximation process of $f^{p}$. In the view of image restoration, based on the (1), to recover $f^{\text {A }}$ from $I^{p}, f^{p}$ gan be sparsely represented by solving the problem:

$$
\int \tilde{\alpha} \overline{\bar{Z}} \arg \min _{\alpha}\left\{\left\|f^{p}-\Delta_{\Gamma} \cdot(h * \phi \alpha)\right\|_{2}^{2}+\beta\|\alpha\|_{1}\right\}
$$

It is expected that $\tilde{g}$ could be close enough to $\alpha$. But due to the degradation factors, especially the aliasing the restoration task is very challenging. Few sparse representation methods fully considered the aliasing, or rather analyzed the aliasing during their processing. Here, we employ the aliasing analysis tool, i.e., adaptive reciprocal cell, to enhance the performance-or sparse representation for image recovery.

We can rewrite the (7) as follow:

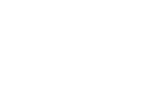

$$
\tilde{\alpha}=\arg \min _{\alpha}\left\{\left\|F\left(f^{p}\right)-F(h * \phi \alpha)\right\|_{\Omega_{a r c}}^{2}+\beta\|\alpha\|_{1}\right\}
$$

where $F($.$) stands for Fourier Transform. In(8), the data-fitting term is defined on the$ support region of adaptive reciprocal cell so that the model can effectively describe the aliasing.

\section{Experimental Results}

We applied restoration method to both simulated corrupted image and real CBERS-02B satellite image. In the simulated image restoration, a blur kernel, i.e. Gaussian function with standard deviation 2 is used. Then, Additive Gaussian noise with level 2 is added to the 
blurred image. After this, we the processed image is down sampling. The basic parameter setting of our proposed method is as follows: the patch size is $9 \times 9$ and $K=120$.

In the experiment, the proposed method is compared with the TV model. As show in Figure 1.

Figure 1(a) is original image,(b) is degraded image, (c) is the image which is record by adaptive Reciprocal Cell, (d) is the restoration result of TV model and (e) is our method. We can see that the result of our method is clear and natural. We can also see the good performance of our method in Figure 2. In Figure 3, we use our method to process the real image, i.e. the CBERS-2B Satellite Image. We can see that our method can achieve good resotred image.

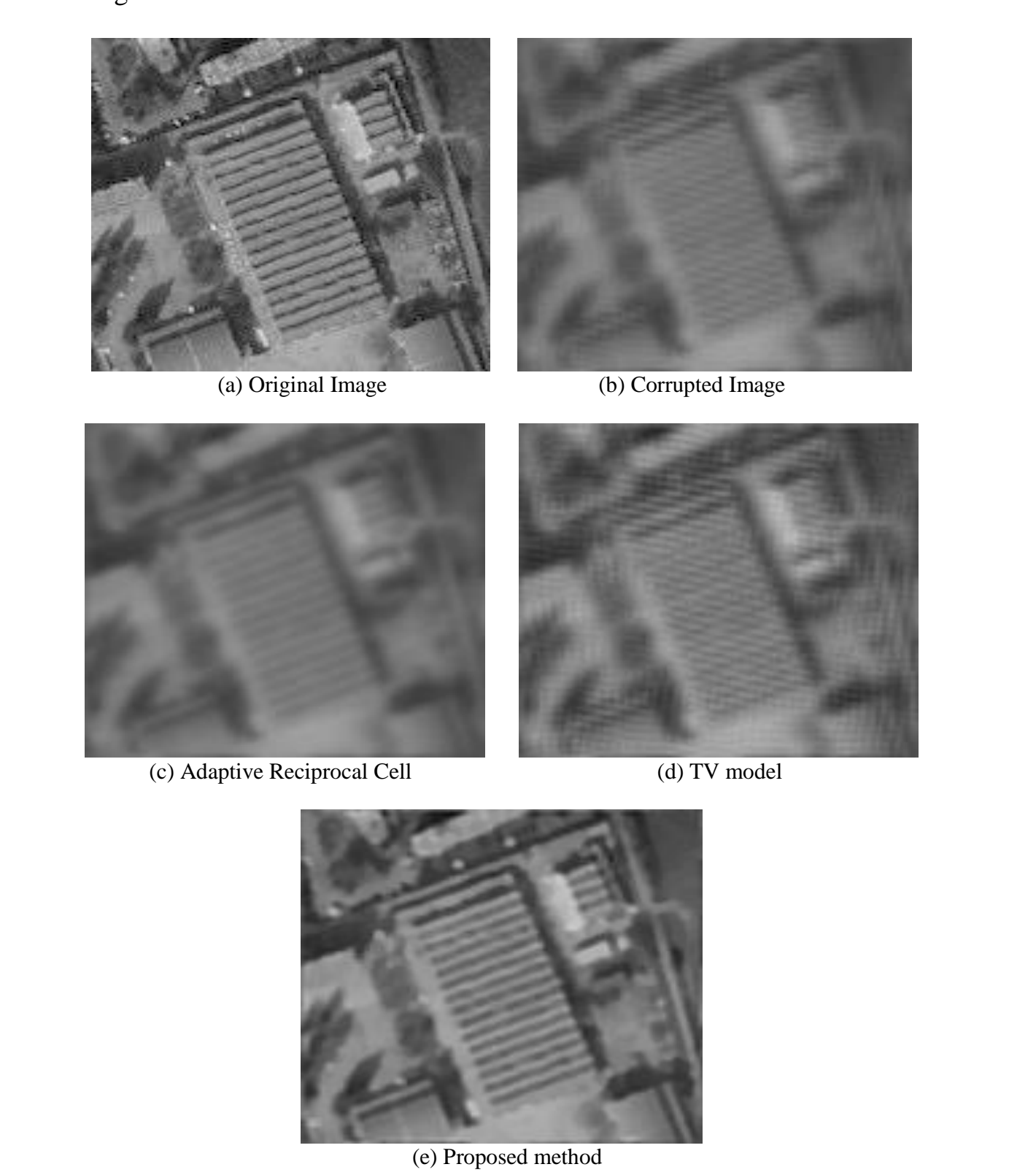

Figure 1. Simulated Image Restoration 


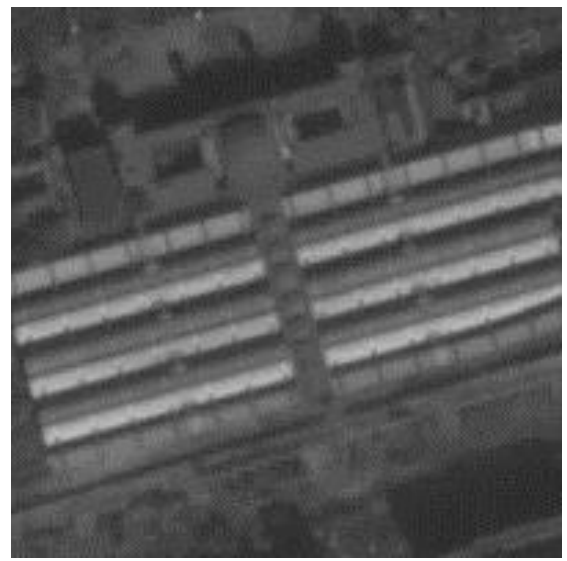

(a) Original Image

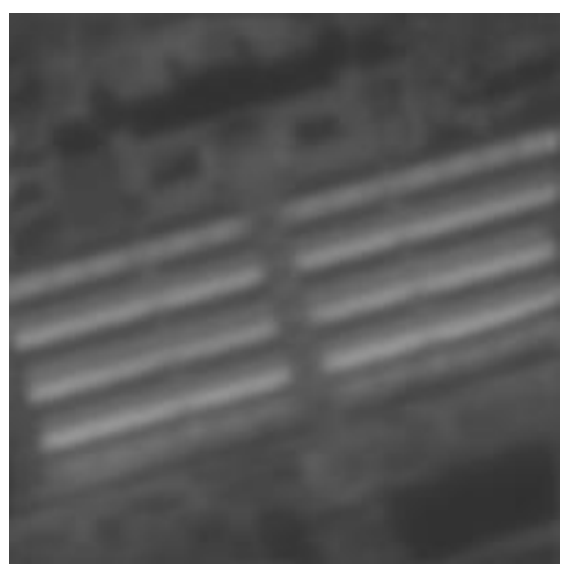

(c) Adaptive Reciprocal/Cell

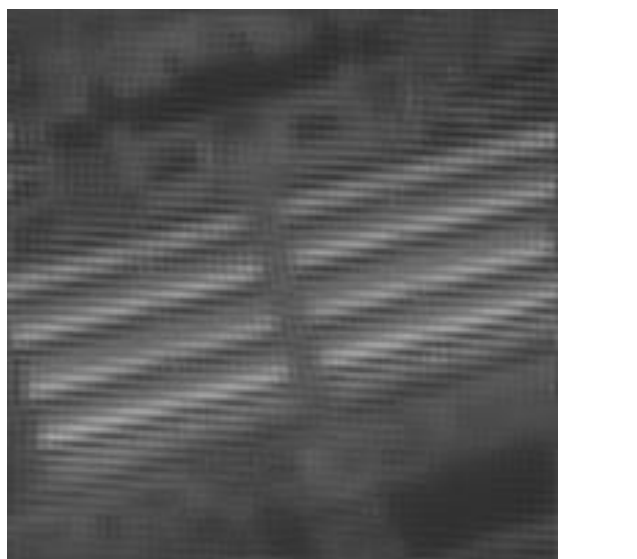

(b) Corrupted Image

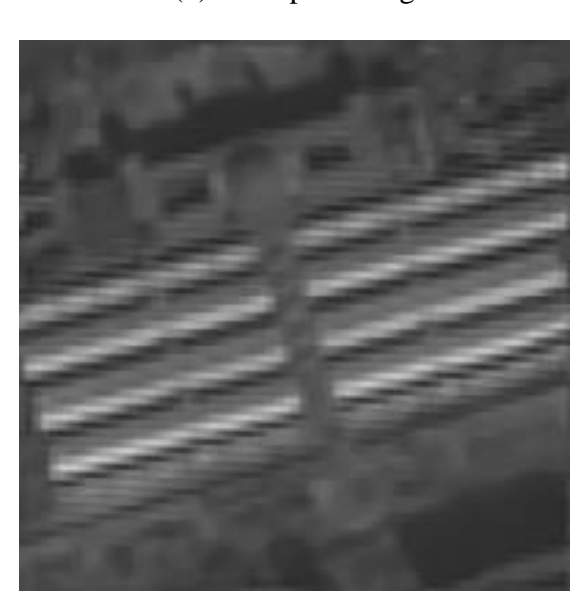

(d) TV model

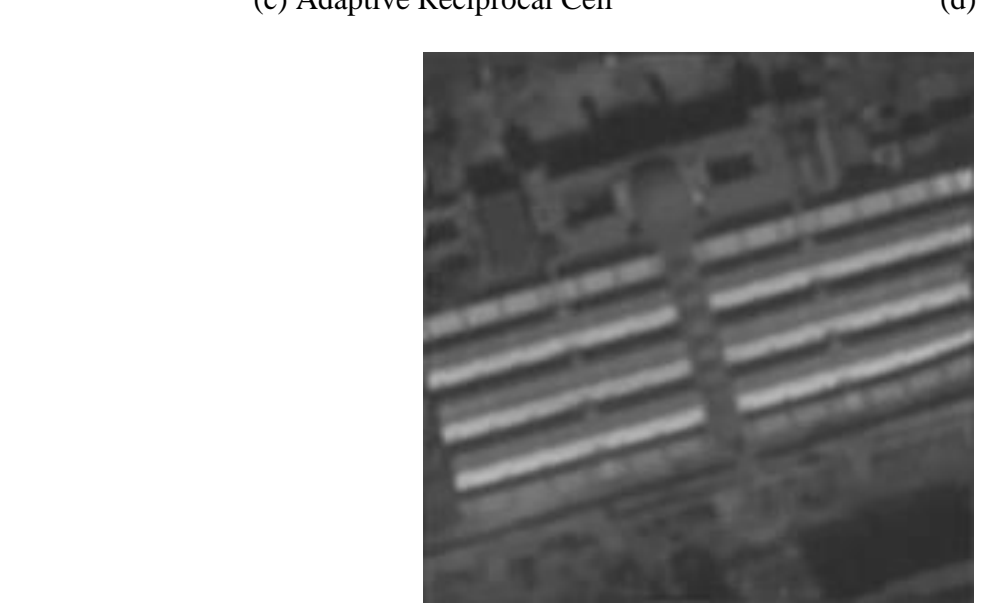

(e) Proposed method

Figure 2. Simulated Image Restoration 


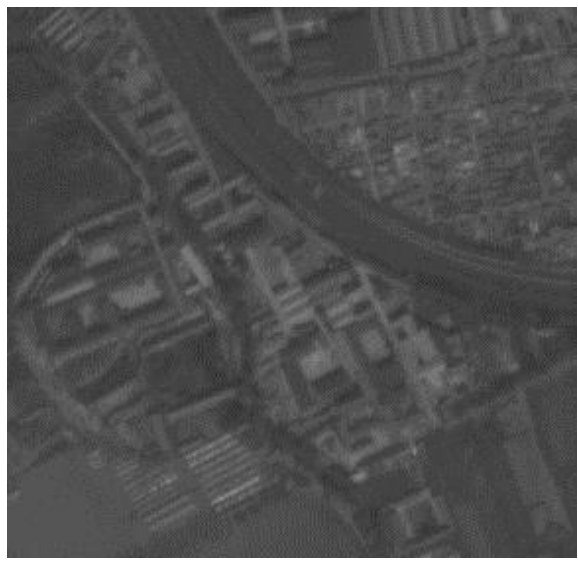

(a)Real satellite image

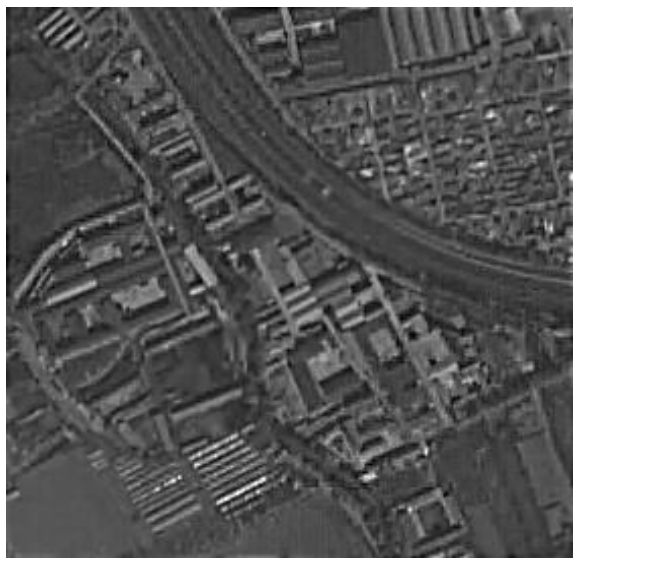

(b) Proposed method

Figure 3. CBERS-2B Satellite Image Restoration

\section{Discussions}

In imaging process, high resolution satellite imáges are tisually unavoidably corrupted by three degradation factors including blur, noise-and aliasing. The satellite image restoration is an ill-posed inverse problem, which requires regularization to avoid unstable solutions. We have proposed a new sparse representation method for satellite image restoration by using the adaptive reciprocal cell to estimate the quality of the degraded image and then introducing it to restoration process in order to avoid amplifying noise. Experiment results show that our method can produce appealing results in vision.

\section{Acknowledgments}

This paper is a revised and expanded version of a paper entitled "Adaptive Reciprocal Cell based Sparse Representation for Satellite Image Restoration" presented at SoftTech 2014, Yeosu, Korea, May 8-10,2014. This research was supported in part by National Natural Science Foundation of China $(61173072,61273251,61402234,61402235)$, the Project Funded by the Priøity Academic Program Development of Jiangsu Higher Education Institutions, Natural Science Foundation of Jiangsu Province (BK2011824, BK2011825), the Innovation Fuńd of China Academy of Space Technology (CAST201302), and China Meteorological Administration under CMA grants (2014 M43).

\section{References}

[1] A. Andres, C. Vicent, H. Gloria and R. Bernard, "Restoration and zoom of irregularly sampled blurred and noisy images by accurate total variation minimization with local constraints", Multiscale Modeling \& Simulation, vol. 51, no. 1, (2006).

[2] D. Tuia and V. G. Camps, "Recent advances in remote sensing image processing", IEEE International Conference on Image Processing, (2009) November 7-9, Cairo, Egypt.

[3] B. Xie and B. L. Jiao, "The PSF estimation method and image restoration algorithm based on Image-Motion analysis of spaceborne TDICCD camera", Journal of Astronautics, vol. 31, no. 3, (2010).

[4] F. Malgouyres, "A framework for image deblurring using wavelet packet bases", Applied and Computational Harmonic Analysis, vol. 12, no. 3, (2002).

[5] A. Jalobeabu, L. Blanc-Feraud and J. Zerubia, "satellite image deblurring using complex wavelet packets", International Journal of Computer Vision, vol. 51, no. 3, (2003). 
[6] R. Neelamani, Choi Hyeokho and R. Baraniuk, "ForVaRD: Fourier-wavelet regularized deconvolution for illconditioned systems", IEEE Transactions on Signal Processing, vol. 52, no. 2, (2004).

[7] A. Buades, B. Coll and J. M. Morel, "A review of image denosing algorithms, with a new one", Multiscale Modeling \& Simulation, vol. 4, no. 2, (2005).

[8] K. Dabov, A. Foi, V. Katkovnik and K. Egizarian, "Image denoising by sparse 3-D transform-domain collaborative filtering", IEEE Transaction on Image Processing, vol. 16, no. 8, (2007).

[9] T. Peleg, Y. Eldar and M. Elad, "Exploiting statistical dependencies in sparse representations for signal recovery", IEEE Transactions on Signal Processing, vol. 60, no. 5, (2012).

[10] F.Malgouyre and F.Guichard, "Edge direction preserving image zooming: a mathematical and numerical analysis", SIAM Journal on Numerical Analysis, vol. 39, no. 1, (2001).

[11] C. R. Vogel and M. E. Oman, "Iterative methods for total variation denoising", SIAM Journal on Scientific Computing, vol. 17, no. 1, (1996).

[12] L. X. Zhu and D. S. Xia, "Staircase effect alleviation by coupling gradient fidelity term", Image and vision computing, vol. 26, no. 8, (2008).

[13] A. A. Zohair, M. Dzulkifli, S. M. R. Mohd and S. L. Ghazali, "Restoring degraded astronomy mages using a combination of denoising and deblurring techniques", International Journal of Signal and Image Processing, vol. 5, no. 1, (2012).

[14] P. Telagarapu, V. Jagan Naveen, A. L. Prasanthi and G. Vijaya Santhi, "Image Compression Using DCT and Wavelet Transformations", International Journal of Signal and Inage Processing, vol, 4, no. 3, (2011).

[15] M. Elad, M. Aharon and A. M. Bruckstein, "K-SVD. An algorithm for designining of overcomplete dictionaries for sparse representation”, IEEE Transactions on Signal Processing, vol. 54, no. 11, (2006).

[16] J. Mairal, M. Elad and G. Sapiro, "Sparse representation for color image restoration", IEEE Transactions on image processing, vol. 17, no. 1, (2008).

[17] W. S. Dong, L. Zhang, G. G. Shi and X. eLi, Nonlocally centralized sparse representation for image restoration", IEEE Transactions on Image Processing, vol. 22, no. 4, (2013).

[18] A. Andres, D. Sylvain and R. Bernard, "Measurng and improving image resolution by the adaptation of the reciprocal cell", Journal of Mathematical Imaging and Vision, yol. 22, no. 3, (2004).

[19] S. Zolta, B. Poczos and A. L. Lorincz, "Online Group Structured Dictionary Learning, IEEE International Conference on Computer Vision and Pattern Recognition", (2011) June 20-25, Colorado Springs, USA.

[20] A. Chambolle, "An algorithm for total variation minimization and applications", Journal of Mathematical imaging and vision, vol. 20, (2004), pp. 1-2.

[21] A. Chambolle, "Image Recovery via Totar Variational Minimization and Related Problems", Numerische Mathematik, vol. 76, no.2, (1997).

[22] J. Yang, J. Wright T. Huang and Ma, "Image super-resolution via sparse representation", IEEE Transactions Image Processing, vol. 19, nd. 11, (2010).

[23] M. Elad and I. Yavneh, "A plurality of sparse representations is better than the sparsest one alone", IEEE Trans.Image Process, vol.55, po. 10, (2009).

[24] J. Mairal, F. Bach, J. Ponce, G. Sapiro and A. Zisserman, "Non-local sparse models for image restoration", IEEE International Conference on Computer Vision, (2009) September 27-October 4, Kyoto, Japan.

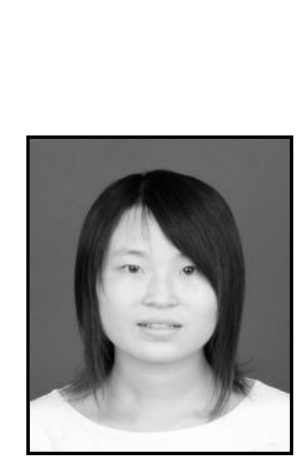

\section{Authors}

Yanfei He, she student at the School of Mathematics and Physics, Nanjing University of Information Science and Technology. Her research interest covers image processing.

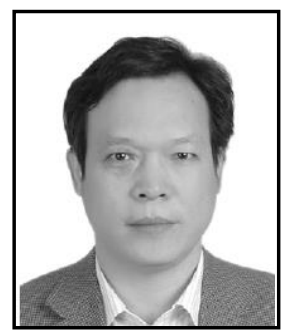

Jianwei Zhang, he is a Professor at the College of Mathematics and Physics, Nanjing University of Information Science and Technology. His research interest covers pattern recognition, artificial intelligence, and remote sensing information processing. Corresponding author of this paper. 


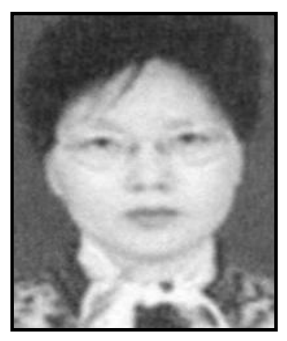

Shunfeng Wang, she is a Professor at the School of BinJiang, Nanjing University of Information Science and Technology. His research interest covers image processing, pattern recognition.

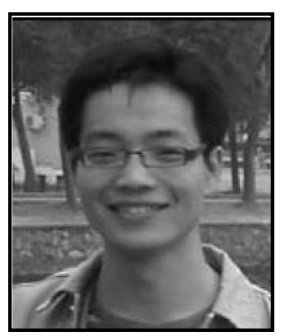

Yuhui Zheng, he is an Associate professor at the School of Computer and Software, Nanjing University of Information Sclence and Technology. His research interest covers image processing, pattern recognition, and remote sensing image restoration.
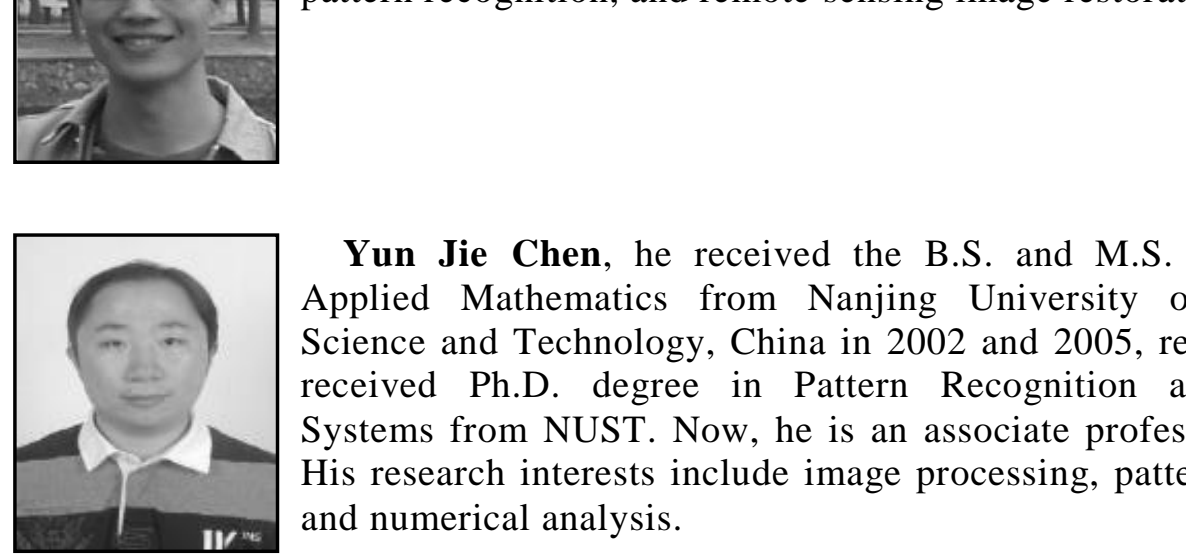

Yun Jie Chen, he recerved the B.S.' and M.S. degree in the Applied Mathematics from Nanjing University of Information Science and Techndogy, China 2002 and 2005, respectively. He received Ph.D. degree in Pattern Recognition and Intelligent Systems from NUST. Now, he is an associate professor in NUIST. His research ihterests include image processing, pattern recognition and numerical analysis.

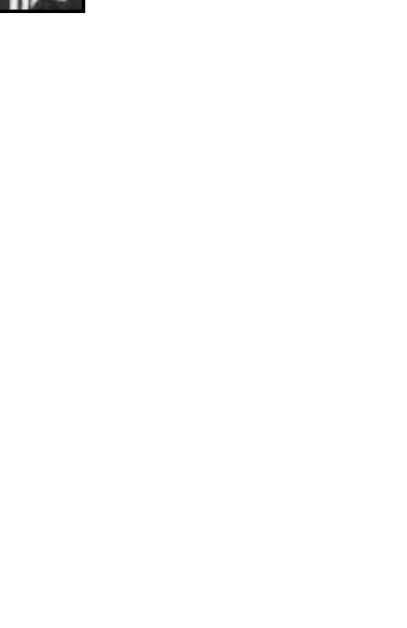

